\title{
Influencia de variables organizacionales en la calidad de vida laboral de funcionarios del sector público de salud en el extremo norte de Chile*
}

Influence of Organizational Variables in Quality of Working Life of Officials Public Health Sector in the Far North of Chile

Recibido: enero 1 de 2013 | Revisado: abril 3 de 2013 | Aceptado: junio 3 de 2013

\author{
DANIEl PÉREZ-ZAPATA ** \\ JenNifer Peralta-Montecinos \\ PAULA FERNÁNDEZ-DÁVILA \\ Universidad de Tarapacá, Arica, Chile
}

doi:10.11144/Javeriana.UPSY13-2.ivoc

Para citar este artículo: Pérez-Zapata, D., PeraltaMontecinos, J. \& Fernández-Dávila, P. (2014). Influencia de variables organizacionales en la calidad de vida laboral de funcionarios del sector público de salud en el extremo norte de Chile. Universitas Psychologica, 13(2), 541-551. doi:10.11144/Javeriana. UPSY13-2.ivoc

\footnotetext{
Artículo de investigación

** Departamento de Filosofía y Psicología, Programa de Magíster en Ciencias Sociales Aplicadas. Correspondencia: Universidad de Tarapacá, Depto. de Filosofía y Psicología. Fax: 56-58-205662. 18 de Septiembre 2222. Arica, Chile. E-mail: daniel. perez.zapata@gmail.com
}

RESUMEN

En el presente estudio se describe la calidad de vida laboral y su relación con el clima organizacional, la satisfacción laboral y el engagement en funcionarios de organizaciones públicas de salud de la ciudad de Arica, Chile. Participaron 142 sujetos de ambos sexos. Se utilizaron el Inventario de Calidad de Vida Laboral, el Inventario de Clima Organizacional, el Cuestionario de Satisfacción Laboral S21/26 y el Cuestionario de Engagement. Los resultados muestran una relación significativa entre la calidad de vida laboral y las variables organizacionales medidas, observando la presencia de diferencias significativas en función del estamento administrativo de los funcionarios. Conjuntamente, se encontró que la variable engagement modula la relación entre la calidad de vida laboral, clima organizacional y satisfacción laboral. Estos resultados evidencian la asociación entre las variables organizacionales e interpersonales que confluyen en el ámbito laboral con las valoraciones subjetivas que los sujetos construyen sobre las características de su entorno de trabajo, modulando su implicación y productividad.

Palabras claves

Calidad de vida laboral, clima organizacional, satisfacción laboral y engagement.

\section{A B S T R A C T}

We describe the Quality of Working Life and its relationship with the Organizational Climate, Job Satisfaction and Engagement in officials of public health organization in the city of Arica, Chile. 142 adult subjects of both sexes. We used the Inventory of Quality of Work Life, Organizational Climate Inventory, Job Satisfaction Questionnaire S21/26, and Engagement Questionnaire. The results show a significant relationship between the quality of working life and organizational variables measures, noting the presence of significant differences in the Quality of Working Life in terms of establishment of civil administration. Jointly, we found that the variable Engagement modulates the relation between Quality of Working Life, organizational climate and job satisfaction. These results demonstrate the association between organizational and interpersonal variables that come together in the workplace, with subjective assessments that subjects build on the characteristics of their work environment, modulating their involvement and productivity.

Keywords

Quality of work life, organizational climate, job satisfaction, engagement. 


\section{Introducción}

La investigación en el ámbito de la psicología laboral se centra actualmente en el estudio del bienestar subjetivo de los trabajadores en su entorno laboral como medida de éxito de los objetivos productivos definidos por las organizaciones (Gómez, 2010; Michie \& Williams, 2003). En este contexto, la medición de la calidad de vida laboral definida como 'la experiencia personal de los empleados respecto a su implicación en el ejercicio de una ocupación', cobra fuerte relevancia debido a su naturaleza multidimensional que concilia los objetivos organizacionales y la experiencia psicológica de los trabajadores, asociándose fuertemente a la satisfacción, motivación y rendimiento laboral (Segurado \& Argulló, 2002).

La calidad de vida laboral se constituye como un concepto subjetivo que abarca todas aquellas condiciones productivas e interpersonales que definen el trabajo, influyendo en el desenvolvimiento psicológico de los individuos en su entorno laboral, junto con la contribución a la capacidad de adaptación, creatividad y voluntad que exigen los objetivos productivos de la organización (Chan \& Wyatt, 2007). En relación con lo anterior, existe evidencia que aquellas organizaciones que desarrollan un entorno de trabajo participativo, ejercen una importante influencia en la satisfacción que sus empleados pudieran presentar, afectando positivamente su salud física, psicológica y emocional (Da Silva, 2006; Gómez, 2006). Conjuntamente, existe evidencia de que las organizaciones que ofrecen oportunidades de desarrollo profesional y personal para sus trabajadores, obtienen mayores ganancias y mayor consideración social (Harter, Schmidt \& Keyes, 2002).

El ejercicio de una ocupación implica el despliegue de recursos organizacionales, relacionales y psicológicos, para satisfacer las distintas demandas derivadas del ejercicio de una ocupación, con el objetivo de lograr un equilibrio entre la organización, el puesto de trabajo y los trabajadores que contribuya a alcanzar los objetivos organizacionales y la adaptación de los trabajadores a los medios de producción (Alonso, 2008; Oliver, Tomás \& Chey- ne, 2005; Salanova \& Llorens, 2009). En relación con lo anterior, en los últimos años surge un gran interés por estudiar el bienestar subjetivo de profesionales del área de la salud, debido a las complejas condiciones organizacionales y relacionales bajo las cuales desarrollan su labor, caracterizándose por la presencia de una alta sobrecarga laboral y escasas redes de apoyo social en el contexto laboral (Khani, Jaafarpour \& Dyrekuandmogadam, 2008; Michie $\&$ Williams, 2003).

Estudios realizados respecto a calidad de vida laboral en funcionarios del área de la salud señalan la presencia de una baja percepción de la misma, junto a una pobre motivación intrínseca, lo que repercute en las fortalezas y debilidades de los ambientes de trabajos (Khani et al., 2008; Paredes \& Paravic, 2006; Shu \& Kernohan, 2006). Por su parte, existe evidencia de que la presencia de variables de gestión como sobrecarga laboral, salario y escases de tiempo disponible influyen desfavorablemente en la sensación de bienestar experimentada por los trabajadores, afectando directamente su rendimiento laboral y su estado de salud, al asociarse a la presencia de altos niveles de ansiedad, los cuales pueden llegar a interferir en la capacidad de adaptación de los sujetos a las distintas exigencias laborales (GarcíaRamos, Luján-López \& Martínez-Corona, 2007; Riveros, Berné \& Múgica, 2010).

Así, la consideración de las condiciones organizacionales y relacionales en que se desarrolla una ocupación resulta crucial para la identificación y abordaje de aquellas variables organizacionales e individuales que contribuyen al bienestar subjetivo de los trabajadores (Abdallah, Thompson, Michaelson, Mark \& Stever, 2009; Caballero \& Blanco, 2007; Gómez, 2010). Con base en lo expuesto, es de suma importancia destacar la influencia de la calidad de las relaciones interpersonales en el contexto laboral y las percepciones que los trabajadores construyen sobre su propio rol, debido al fuerte impacto que generan en la satisfacción de sus necesidades y en la motivación y compromiso hacia la ocupación, al actuar como agentes mediadores para el bienestar subjetivo, existiendo evidencia de que el clima organizacional, satisfacción laboral y engagement se consti- 
tuyen en importantes factores predictores para la percepción de calidad de vida laboral (Harter et al., 2002; Salanova \& Llorens, 2008; Van Laar, Edwards \& Easton, 2007).

El clima organizacional es definido como 'el conjunto de percepciones que los trabajadores construyen respecto a las estructuras y procesos que se producen en un medio laboral', comprendiendo aquellas particularidades que distinguen a su organización de otras y constituyéndose así en un importante factor para la consecución de los objetivos organizacionales al modular las actitudes y conductas de los trabajadores (Chiang, Salazar \& Núñez, 2007). Respecto a su asociación con la calidad de vida laboral, existe evidencia que el clima organizacional influye en esta, a través de la motivación intrínseca de los trabajadores hacia el desarrollo de su ocupación, que se deriva de la percepción de cohesión grupal y apoyo directivo (Casas, Repullo, Lorenzo \& Cañas, 2002; MuñosSeco, Coll-Benejam, Torrent-Quetglas \& LinaresPou, 2006; Ramírez \& Zurita, 2010).

Hallazgos de estudios realizados sobre clima laboral en organizaciones de salud señalan una alta percepción de liderazgo y participación, no obstante, existe evidencia de una pobre percepción de los trabajadores sobre algunas dimensiones del clima organizacional correspondientes a responsabilidad, aplicación al trabajo y compromiso con la productividad, las que afectan significativamente los niveles de motivación de los trabajadores hacia el desarrollo de su ocupación, mermando directamente su desempeño (Pérez, Soler \& Díaz, 2009; Santa Eulalia \& Sampedro, 2012).

Respecto a la presencia de satisfacción laboral, esta es definida como 'una actitud o disposición para actuar de un modo determinado en relación con aspectos específicos del trabajo y la satisfacción con el mismo' (Sirgy, Efraty, Siegel \& Lee, 2001; Van Laar et al., 2007). La inclusión de esta variable en la medición de la calidad de vida laboral se debe principalmente a que la satisfacción laboral influye directamente en la calidad del trabajo realizado, constituyéndose en un elemento estructural de la organización que favorece la productividad (Acker, 2004).
Estudios realizados respecto a la presencia de Satisfacción Laboral en funcionarios del área de la salud indican que esta se explica por la interrelación de diversos factores organizacionales e individuales como la satisfacción con el sueldo, la carga laboral, el tipo de prácticas laborales y las características individuales (Katerndahl, Parchman \& Wood, 2009; Navarro-Reynoso, Hernández-Lamas, RobledoGalván \& Romero-Palacios, 2008; Zuger, 2004).

En relación con el engagement, definido como 'un estado mental positivo, de realización, asociado con el trabajo que se caracteriza por vigor, dedicación y absorción' (Schaufeli, Salanova, GonzalezRoma \& Bakker, 2002; Raigosa \& Marín, 2010), este se constituye en un importante constructo motivacional que se caracteriza por la energía que muestra el trabajador en su ocupación, que no se asocia a una situación concreta o temporal dentro del ámbito laboral ni a un modo particular de comportamiento, sino, más bien, a un estado cognitivoafectivo prolongado que se deriva de experiencias placenteras en las esferas física, psicológica y emocional (Salanova \& Llorens, 2009; Schaufeli \& Bakker, 2004). La presencia de engagement en los trabajadores de una organización se asocia significativamente con la consecución de una calidad de vida laboral positiva, al ejercer un rol modulador en otras variables organizacionales que coexisten dentro del ámbito laboral como el estilo de liderazgo, la cultura organizacional y el clima organizacional (Ortiz \& Arias, 2009; Salanova \& Llorens, 2008).

La diversidad de variables organizacionales e interpersonales que confluyen en el ámbito laboral, asociándose a las valoraciones subjetivas que los sujetos pudieran realizar sobre las características de su entorno de trabajo, así como de su implicación y desempeño laboral, hacen necesaria una indagación respecto a la influencia de dichas construcciones en el bienestar subjetivo de los trabajadores, que guíen el desarrollo de nuevas estrategias organizacionales orientadas al logro de una mayor implicación laboral y al surgimiento de una calidad de vida laboral positiva (Gil-Monte, Carretero \& Roldán, 2005; Ortiz \& Arias, 2009; Salanova \& Llorens, 2009).

Considerando que la cultura organizacional y el medio social pueden establecer diferencias impor- 
tantes en la calidad de vida laboral de los trabajadores, el objetivo de esta investigación es describir la relación entre satisfacción laboral, clima organizacional y engagement con la calidad de vida laboral en funcionarios de instituciones públicas de salud en la ciudad de Arica, Chile. Siendo las hipótesis de estudio las siguientes: 1) a mayor percepción de clima organizacional, mayor calidad de vida laboral en trabajadores; 2) a mayor presencia de satisfacción laboral en los trabajadores, mayor calidad de vida laboral y 3) a mayor presencia de engagement en trabajadores, mayor calidad de vida laboral. Esperando encontrar que la variable engagement ejerce una influencia moduladora entre calidad de vida laboral, clima organizacional y satisfacción laboral, según investigaciones previas.

\section{Método}

\section{Sujetos}

La muestra estudiada correspondió a 142 sujetos adultos, de ambos sexos, cuyas características sociodemográficas se exponen en la Tabla 1.

\section{Instrumentos}

Inventario de Calidad de Vida

Laboral (Da Silva et al., 2006)

Compuesto por 82 ítems, recoge información sobre la percepción y sensación de bienestar que experimentan los trabajadores en su entorno laboral, en once dimensiones: 1) Sistema de Gestión, 2) Relación con Compañeros, 3) Relación con Jefes, 4) Motivación, identificación y compromiso, 5) Condiciones del entorno laboral, 6) Estrés y burnout, 7) Equilibrio trabajo-vida privada, 8) Satisfacción con el Rol y Potencial Motivador del Puesto, 9) Bienestar subjetivo derivado del trabajo, 10) Equidad Remunerativa y 11) Características de Personalidad. Las respuestas corresponden a una escala de acuerdo que abarca desde muy en desacuerdo a muy de acuerdo. El instrumento presenta adecuadas propiedades psicométricas, claridad de sus reactivos y una apropiada consistencia interna, en cada una de las dimensiones con confiabilidades que oscilan entre 0.795 y 0.948 y un $\alpha$ de Cronbach de 0.948 para la escala total.

\section{Escala de Clima Laboral (Ramírez, 2008)}

Su utilidad radica en la valoración de variables interpersonales y organizacionales que influyen en el clima al interior de una organización. Consta de 42 ítems, divididos en 8 dimensiones: 1) Compromiso Extrínseco, 2) Compañeros y Cohesión, 3) Apoyo del Supervisor, 4) Descripción de Cargo, 5) Infraestructura, 6) Equidad y Autonomía, 7) Reconocimiento de Méritos y 8) Compromiso Intrínseco. Su respuesta corresponde a una escala de acuerdo que abarca desde totalmente en desacuerdo a totalmente de acuerdo. El instrumento presenta

TABLA 1

Características sociodemográficas de los participantes

\begin{tabular}{lcc}
\hline Edad & $\begin{array}{c}33.9 \text { años } \\
(\mathrm{DE}=9.3)\end{array}$ & $\begin{array}{c}\text { Sexo \% } \\
(\mathrm{N}=142)\end{array}$ \\
\hline Masculino & 44 & 31 \\
Femenino & 98 & 69 \\
Nivel Educativo & & Porcentaje \\
Enseñanza Básica & 2 & 1.4 \\
Enseñanza Media & 4 & 2.8 \\
Educación Superior & 48 & 33.8 \\
Educación Técnica & 64 & 45.1 \\
Estudios de Postgrado & 20 & 14.1 \\
Organización de Salud & & Porcentaje \\
CESFAM: Sapunar & 61 & 43 \\
CESCOF: Cerro la Cruz & 6 & 4.2 \\
CESFAM: Bertin Soto & 75 & 52.8 \\
Cargos & & \\
Directivos & 10 & 7 \\
Profesionales & 57 & 40.1 \\
Técnicos & 59 & 41.6 \\
Administrativos & 16 & 10.9 \\
Antigüedad Organizacional & & \\
Menos de 2 años & 26 & 19.3 \\
2 a 5 años & 46 & 33.4 \\
6 a 10 años & 23 & 16.2 \\
11 a 15 años & 17 & 10.6 \\
16 a 20 años & 17 & 10.6 \\
Más de 20 años & 13 & 9.5 \\
\hline
\end{tabular}

Fuente: elaboración propia 
adecuadas propiedades psicométricas en sus dimensiones y en la escala total, con confiabilidades que oscilan entre 0.7 y 0.916 para cada dimensión y un $\alpha$ de Cronbach de 0.951 para la escala total.

Escala de Satisfacción Laboral S21/26 (Meliá et al., 1990)

Su utilidad radica en la valoración de la actitud y disposición que los trabajadores presentan hacia el ejercicio de la ocupación. Se compone de 26 ítems divididos en 6 dimensiones: 1) Satisfacción con la supervisión y participación en la organización, 2) Satisfacción con las remuneraciones y prestaciones, 3) Satisfacción Intrínseca, 4) Satisfacción con el Ambiente Físico, 5) Satisfacción con la Cantidad de Producción y 6) Satisfacción con la calidad de producción. La escala de respuesta del instrumento corresponde a una escala de valoración que abarca desde muy insatisfecho a muy satisfecho. La escala presenta adecuadas propiedades psicométricas, mostrando fiabilidades que oscilan entre 0.731 y 0.891 para cada una de las dimensiones y un alfa de Cronbach de 0.931 para la escala total.

Versión en español de la escala Utrecht Work Engagement Scale UWES (Schaufeli Salanova, González-Romá Ė Bakker, 2002)

Su utilidad radica en la valoración de un estado psicológico positivo respecto al trabajo. Se compone de 17 ítems, divididos en 3 dimensiones: Vigor, Dedicación y Absorción. Sus respuestas corresponden a una escala tipo Likert de seis puntos que abarca desde Nunca a Siempre. El instrumento muestra adecuadas propiedades psicométricas, presentando fiabilidades que oscilan entre 0.79 y 0.84 para cada una de las dimensiones, y un alfa de Cronbach de 0.89 para la escala total.

\section{Diseño y Procedimiento}

Se utilizó un diseño no experimental retrospectivo de carácter correlacional con variables intervalares y nominales. Para recolectar los datos, se le entregó a cada uno de los participantes, cuadernillos con los instrumentos descritos arriba, para ser contestados de forma anónima y consignando información sobre variables sociodemográficas generales. El tiempo de respuesta de los cuadernillos fue de una hora en promedio. El análisis de datos se llevó a cabo a través del paquete estadístico para las ciencias sociales (SPSS 15.0). Se efectuó análisis de correlación y análisis de varianza (ANOVA).

\section{Resultados}

Con el objetivo de estudiar la influencia que el clima organizacional, la satisfacción laboral y el engagement ejercen sobre la calidad de vida laboral de los participantes, se correlacionaron las puntuaciones obtenidas en el inventario de calidad de vida laboral con los resultados de las escalas de clima laboral, satisfacción laboral (S21/26) y de engagement (UWES). Se encontraron correlaciones positivas y estadísticamente significativas entre CVL y CO, SL y ENG (Tabla 2).

Para determinar qué dimensiones relacionadas con la escala de clima laboral eran necesarias para explicar la puntuación total en la escala de calidad de vida laboral, se aplicó un análisis de regresión múltiple con el método de pasos sucesivos, en cuya ecuación de regresión entró a formar parte del modelo en primer lugar la dimensión Equidad y Autonomía con un $R=0.632$ que explicaba un $40 \%$ de su varianza, porcentaje significativo con $p \leq 0.001$. En segundo lugar, entró a formar parte de la ecuación la dimensión Infraestructura con un $R=0.669$ que explicaba un $44 \%$ de su varianza, porcentaje significativo con $p \leq 0.001$. En tercer lugar, entró a formar parte de la ecuación la dimensión Compromiso Intrínseco con un $R=$ 0.695 que explicaba un $48 \%$ de su varianza, porcentaje significativo con $p \leq 0.001$. Finalmente, entra a formar parte de la ecuación la dimensión Compañeros y Cohesión, alcanzando $R$ un valor de 0.706 , que explica un $49 \%$ de la varianza de la calidad de vida laboral.

Por su parte, para determinar qué dimensiones relacionadas con la escala de satisfacción laboral eran necesarias para explicar la puntuación total en la escala de calidad de vida laboral, se aplicó 
TABLA 2

Correlaciones generales de calidad de vida laboral con clima organizacional, satisfacción laboral y engagement

\begin{tabular}{lcc}
\hline & & CVL General \\
\hline CO General & Correlación de Pearson & $0.673(* *)$ \\
SL General & Correlación de Pearson & $0.658(* *)$ \\
ENG General & Correlación de Pearson & $00.368(* *)$ \\
\hline
\end{tabular}

** La correlación es significativa al nivel 0.01 (bilateral).

Fuente: elaboración propia

un análisis de regresión múltiple con el método de pasos sucesivos, en cuya ecuación de regresión entró a formar parte del modelo en primer lugar la dimensión Satisfacción con la Supervisión con un $R=0.591$ que explicaba un $35 \%$ de su varianza, porcentaje significativo $\operatorname{con} p \leq 0.001$. En segundo lugar, entró a formar parte de la ecuación la dimensión Satisfacción con el Ambiente Físico con un $R=0.652$ que explicaba un $42 \%$ de su varianza, porcentaje significativo $\operatorname{con} p \leq 0.001$. Finalmente, entra a formar parte de la ecuación de regresión la dimensión Satisfacción con la Remuneración con un $R=0.666$ explicando un $44 \%$ de su varianza.

A su vez, para determinar que dimensiones relacionadas con la escala UWES eran necesarias para explicar la puntuación total en la escala de calidad de vida laboral, se aplicó un modelo de regresión múltiple con el método de pasos sucesivos en cuya ecuación únicamente entró a formar parte la ecuación la dimensión Dedicación con un $R=0.38$ que explicaba un $14 \%$ de su varianza, porcentaje significativo $\operatorname{con} p=0.001$.

Para analizar la existencia de diferencias significativas en la calidad de vida laboral de los participantes en función del cargo, se aplicó un análisis ANOVA de diferencias de medias. En dicho análisis, se obtuvo una diferencia estadísticamente significativa para la variable estamento, $F(3,128)=$ 2.928, $p<0.05$. Así, para determinar la diferencia específica entre los niveles del estamento, se utilizó la prueba post hoc de Bonferroni, la cual mostró que hay diferencias estadísticamente significativas entre el estamento directivo y técnico $(p<0.036)$. Las puntuaciones más altas de CVL corresponden al estamento directivo (Tabla 3).

Para determinar si el ENG funciona como variable moduladora entre CVL y CO, y CVL y SL, se crearon dos grupos en función de sus puntajes medios: engagement bajo y engagement alto. Luego, se utilizó la transformación de Fisher para comparar los coeficientes de correlación para dos muestras

TABLA 3

ANOVA de un factor para calidad de vida laboral general por niveles de estamento y prueba de Bonferroni entre niveles de estamento

\begin{tabular}{lccccccc}
\hline & CVL General & $N$ & Media & DE & Df & $F$ \\
\hline & Directivo & 10 & 305.2 & 48.65 & $3 / 128$ & $2.928^{*}$ & \\
& Profesional & 53 & 280.03 & 31.57 & & & \\
& Técnico & 55 & 269.85 & 38.56 & & & \\
& Administrativo & 14 & 272.64 & 33.22 & & & \\
\hline Directivo & Sig. & Profesional & Sig. & Técnico & Sig. & Administrativo & Sig. \\
\hline Profesional & 0.27 & Directivo & 0.27 & Directivo & $0.03 *$ & Directivo & 1 \\
Técnico & $0.03^{*}$ & Técnico & 0.87 & Profesional & 0.87 & Profesional & 1 \\
Administrativo & 1 & Administrativo & 1 & Administrativo & 1 & Técnico & 1 \\
\hline
\end{tabular}

$* p<0.05$.

Fuente: elaboración propia 


\section{TABLA 4}

Correlaciones entre calidad de vida laboral general, clima organizacional general y satisfacción laboral general, segmentadas de acuerdo a engagement alto y bajo.

\begin{tabular}{ccccc}
\hline CVL General & N & & & \\
\hline Engagement Bajo & SL General & Correlación de Pearson & $0.747(56 \%)$ & 63 \\
& CO General & Correlación de Pearson & $0.752(57 \%)$ & 61 \\
\hline Engagement Alto & SL General & Correlación de Pearson & $0.52(27 \%)$ & 74 \\
& CO General & Correlación de Pearson & $0.557(33 \%)$ & 74 \\
& CVL con SL & Z & 2.22 & \\
& & Significación & $0.02\left(^{*}\right)$ \\
& CVL con CO & Z & 1.99 \\
& & Significación & $0.046\left(^{*}\right)$ \\
\hline
\end{tabular}

Los valores entre paréntesis corresponden a la varianza explicada.

$* p<0.05$.

Fuente: elaboración propia

independientes. En la Tabla 4, se muestran los resultados de las correlaciones. El resultado de la comparación entre los grupos de engagement para CVL y CO fue estadísticamente significativo $(z=$ 1.99, $p<0.046)$. Así mismo, el resultado entre los grupos de engagement para CVL y SL fue estadísticamente significativo $(z=2.22, p<0.02)$.

\section{Discusión}

Considerando que las variables psicosociales presentes en el entorno laboral ejercen un importante rol modulador en la implicación y satisfacción de los trabajadores en el ejercicio de su ocupación, cabe mencionar que los resultados obtenidos en el presente estudio muestran una asociación entre calidad de vida laboral, clima organizacional, satisfacción laboral y engagement, concordando con investigaciones previas realizadas (Baba \& Jamal, 1991; Chan \& Wyatt, 2007; Van Laar et al., 2007).

Lo anterior se debería a que la valoración subjetiva sobre la calidad de vida laboral se encuentra estrechamente asociada a la percepción de los trabajadores respecto a los estilos directivos presentes en su organización, con énfasis en aquellos procesos de interacción que fomentan la autonomía, participación y retroalimentación (Jubete, Lacalle, Riesgos, Cortés \& Mateo, 2005). En relación con lo expuesto, la presencia de estilos cohesionados de participación en el ámbito laboral influye po- sitivamente en la motivación de los trabajadores hacia el ejercicio de su ocupación, al percibir mayor apoyo del equipo y una mayor adaptación a las demandas laborales, contribuyendo directamente en la satisfacción e implicación del personal en las acciones y procesos concernientes a su ocupación, lo que influye en las valoraciones subjetivas que los sujetos pudieran realizar respecto a las condiciones de su empleo y en la calidad de los servicios entregados (Álvarez-Nido, Sánchez-González \& Lorenzo-Borda, 2006; Muñoz-Seco et al., 2006).

En referencia a las dimensiones especificas de las variables psicosociales medidas que influyen en la calidad de vida laboral de los participantes del estudio, cabe mencionar que respecto al clima organizacional, la percepción de los trabajadores respecto a la adecuación de la infraestructura del lugar de trabajo, la equidad y autonomía en el desarrollo de sus funciones y la presencia de compromiso intrínseco, cohesión y compañerismo, se asocian a una mayor percepción de calidad de vida laboral, al relacionarse con una mayor adaptación a las demandas laborales derivada de una disminución en la sobrecarga laboral, resultado del trabajo participativo e integrado (Muñoz-Seco et al., 2006).

Por su parte, respecto a la satisfacción laboral, la presencia de factores remunerativos, de interacción con pares y de supervisión de las actividades realizadas se asocian a una mayor valoración de calidad de vida laboral al relacionarse con la calidad 
de las relaciones interpersonales que se establecen en el trabajo, las que se constituyen en importantes fuentes de apoyo y dirección para el ejercicio de la ocupación y a la disposición de medios económicos que permiten satisfacer las distintas necesidades personales de los trabajadores (Fernández \& Paravic, 2003; Paris \& Omar, 2008; Robles-García et al., 2005). Por último, en referencia al engagement, la presencia de dedicación en el trabajo se asocia a una mayor percepción de calidad de vida laboral en el personal, debido a su relación con la presencia de facilitadores para el puesto de trabajo que generan en los trabajadores mayores expectativas de autoeficacia (Raigosa \& Marín, 2010; Salanova \& Schaufeli, 2004).

En referencia a la existencia de diferencias significativas en la percepción de calidad de vida laboral en función del cargo de los participantes, se observa que existen diferencias en las valoraciones subjetivas entre los sujetos con cargos directivos y técnicos (García-Ramos et al., 2007; Riveros et al., 2010). Respecto a lo anterior, cabe señalar que estas diferencias podrían ser explicadas por la estructura organizacional de los servicios de salud pública de Chile, los cuales en sus estamentos técnicos, se conforman mayoritariamente por profesionales técnicos paramédicos, los cuales se encuentran expuestos a una alta demanda laboral, ejecutando estandarizada y rutinariamente sus labores con un contacto permanente con el usuario de salud, conjuntamente, deben realizar sus funciones con la provisión de escasos insumos asistenciales y escasas comodidades en infraestructura, lo que se asociaría a una pobre percepción de calidad de vida laboral. en relación a lo anterior, sería importante considerar que la diferencia de los resultados entre el estamento directivo y técnico, se podría deber a los niveles altos de inequidad percibida por parte del estamento técnico. La percepción de inequidad en los intercambios sociales es una fuente de estrés que influye de manera negativa en los niveles de realización personal en el trabajo y en la valoración de la calidad de vida laboral (Gil-Monte et al., 2005; Taris, Peeters, Le Blanc, Schreurs \& Shaufeli, 2001).

En relación al rol modulador que ejerce la variable engagement en la relación entre calidad de vida laboral, clima organizacional y satisfacción laboral, los hallazgos señalan que respecto a la relación entre calidad de vida laboral y clima organizacional, aquellas personas que experimentan altos niveles de engagement poseen altos niveles de implicación, dedicación y absorción con su trabajo, por lo que la percepción de calidad de vida laboral y de clima organizacional disminuyen frente a esta condición, lo cual puede ser ocasionado porque ambas variables están compuestas, entre otras, por factores externos o del entorno laboral (Bakker, Schaufeli, Leiter \& Taris, 2008; Salanova \& Schaufeli, 2008). Por su parte, para la relación entre calidad de vida laboral y satisfacción laboral los resultados indican que aquellos funcionarios que poseen niveles bajos de engagement, desarrollan un menor compromiso con el trabajo. a su vez, la presencia de altos niveles de engagement en los trabajadores se relacionan con mayores niveles de bienestar subjetivo y una mayor motivación y compromiso hacia la ocupación (Salanova \& Llorens, 2008, 2009; Salanova, Shaufeli, Llorens, Peiró \& Grau, 2000). Estos resultados se constituyen en un antecedente para la investigación que se desarrolla en el campo de las organizaciones, debido a los escasos antecedentes que constatan el rol modulador del engagement en variables psicosociales y, particularmente, sobre la calidad de vida laboral (De Lange, De Witte \& Notelaers, 2008; Fourie, Rothmann \& Van de Vijver, 2008).

Los resultados obtenidos en el presente estudio, pueden entregar nuevos aportes sobre el estado de la calidad de vida laboral en organizaciones del área de la salud, considerando la existencia de múltiples riesgos psicosociales que pueden afectar el bienestar de sus integrantes (Peiró, 2004). Cabe enfatizar la diferencia encontrada entre el estamento técnico y directivo respecto a su percepción de calidad de vida laboral, debido a su asociación con la percepción de inequidad organizacional, orientando nuevos lineamientos y pautas de de acción en la estructura organizacional de los servicios de salud (Gómez, 2006; Pozo, Alonso, Hernández \& Martos, 2005). En relación con lo anterior, estos hallazgos pueden constituirse en nuevos precedente para los equipos directivos de las instituciones de salud, incorporando nuevas decisiones que apunten al me- 
joramiento de la calidad de vida laboral al interior de la organización, principalmente, porque (a) el mejoramiento de los ambientes laborales y sociales influyen directamente en el bienestar subjetivo de sus funcionarios y (b) porque son éstos los principales responsables de entregar servicios de salud de calidad a la población de un país. (Barrios \& Paravic, 2009; Paredes \& Paravic, 2006).

Una interrogante que sería interesante de explorar en futuros estudios sobre calidad de vida laboral en funcionarios de salud, es sí existen diferencias entre organizaciones de salud públicas y privadas. Los resultados que se desprendan de éstas interrogantes, podrían constituirse en importantes parámetros para evaluar diferencias y similitudes en la situación de la calidad de vida laboral en las instituciones de salud a nivel nacional. En cuanto a las limitaciones de este estudio, éstas se relacionan con (a) la posible fatiga experimentada por los funcionarios de salud debido a la extensión de los cuestionarios que debieron contestar y (b) con el diseño del estudio, pudiendo resolverse esta dificultad utilizando un diseño cuasi experimental, sin embargo, con frecuencia es una situación que acompaña a este tipo de trabajos. Finalmente, el presente estudio es un aporte para la investigación en calidad de vida laboral y la relación que ésta tiene con otras variables psicosociales, debido a los escasos estudios realizados en el contexto local.

\section{Referencias}

Abdallah, S., Thompson, S., Michaelson, J., Mark, N., \& Stever, N. (2009). The happy planet index 2.0. London: New Economics Foundation.

Acker, G. M. (2004). The effect of organizational conditions (role conflict, role ambiguity, opportunities for professional development, and social support) on job satisfaction and intention to leave among social workers in mental health care. Community Menthal Health, 40(1), 65-73.

Alonso, P. (2008). Estudio comparativo de la satisfacción laboral en el personal de administración. Psicología del Trabajo y de las Organizaciones, 24(1), 25-40.

Álvarez-Nido, R., Sánchez-González, R., \& LorenzoBorda, M. S. (2006). Consideraciones sobre la calidad de vida profesional en los trabajadores de atención primaria de Madrid. Aten Primaria, 37(5), 305-306.

Baba, V. V., \& Jamal, M. (1991). Routinisation of job context and job content as related to employees quality of working life: A study of psychiatric nurses. Journal of Organizational Behavior, 12(1), 379-386.

Bakker, A., Schaufeli, W., Leiter, M., \& Taris, T. (2008). Work engagement: An emerging concept in occupational health psychology. Work $\mathcal{E}$ Stress, 22(3), 187-200.

Barrios, S., \& Paravic, T. (2009). Percepción de violencia en usuarios hospitalizados en los servicios clínicos de un hospital público de la Región de la Araucanía de Chile. Ciencia y Enfermería, 15(3), 29-43.

Caballero, D., \& Blanco, A. (2007). Competencias para la flexibilidad. La gestión emocional de las organizaciones. Psicothema, 19(4), 616-620.

Casas, J., Repullo, J. R., Lorenzo, S., \& Cañas, J. J. (2002). Dimensiones y medición de la calidad de vida laboral en profesionales sanitarios. Revista de Administración Sanitaria, 6(23), 143-160.

Chan, K. W., \& Wyatt, T. A. (2007). Quality of work life: A study of employees in Shangahi, China. Asia Pacific Business Review, 13(4), 501-507.

Chiang, M., Salazar, C., \& Nuñez, A. (2007). Clima organizacional y satisfacción laboral en un establecimiento de la salud estatal: Hospital tipo 1. Theoria, 16(2), 61-76.

Da Silva, M. (2006). Nuevas perspectivas de la calidad de vida laboral y sus relaciones con la eficacia organizacional. Tesis Doctoral, Universidad de Barcelona, España.

De Lange, A., de Witte, H., \& Notelaers, G. (2008). Should I stay or should I go? Examining longitudinal relations among job resources and work engagement for stayers versus movers. Work $\mathfrak{E}$ Stress, 22(3), 201-223.

Fernández, B., \& Paravic, T. (2003). Nivel de satisfacción laboral en enfermeras de hospitales públicos y privados de la provincia de Concepción, Chile. Cienc enferm, 9(2), 57-66.

Fourie, L., Rothmann, S., \& Van de Vijver, F. (2008). A model of work wellness for non professional counselors in South Africa. Stress $\mathcal{E}$ Health: Jour- 
nal of the International Society for the Investigation of Stress, 24(1), 35-47.

García-Ramos, M., Luján-López, M. E., \& MartínezCorona, M. A. (2007). Satisfacción laboral del personal de salud. Revista de Enfermería del Instituto de Seguro Social, 15(2), 63-72.

Gil-Monte, P. R., Carretero, N., \& Roldán, M. D. (2005). Algunos procesos psicosociales sobre el síndrome de quemarse por el trabajo (burnout) en profesionales de enfermería. Ansiedad y Estrés, 11(2-3), 281-290.

Gómez, I. C. (2006). Salud laboral: una revisión a la luz de las nuevas condiciones del trabajo. Universitas Psicológica, 3(2), 179-186.

Gómez, M. A. (2010). Calidad de vida laboral en empleados temporales del valle de Aburrá-Colombia. Revista Ciencias Estratégicas, 18(24), 225-236.

Harter, J. K., Schmidt, F. L., \& Hayes, T. L. (2002). Business unit-level relationship between employee satisfaction, employee engagement, and business outcomes: A meta-analysis. Journal of Applied Psychology, 87(2), 268-279.

Jubete, M. T., Lacalle, M., Riesgos, R., Cortés, J. A., \& Mateo, C. (2005). Estudio de la calidad de vida profesional en los trabajadores de atención primaria del área 1 de Madrid. Aten Primaria, 36(2), 112-114.

Khani, A., Jaafarpour, M., \& Dyrekuandmogadam, A. (2008). Quality of nursing work life. Journal of Clinical and Diagnostic Research, 2(6), 1169-1174.

Katerndahl, D., Parchman, M., \& Wood, R. (2009). Perceived complexity of care, perceived autonomy, and career satisfaction among primary care physicians. Journal of the American Board of Family Medicine, 22(1), 24-33.

Meliá, J. L., Pradilla, J. F., Martí, N., Sancerni, M. D., Oliver, A., \& Tomás, J. M. (1990). Estructura factorial, fiabilidad y validez del Cuestionario de Satisfacción 21/26: un instrumento con formato dicotómico orientado al trabajo profesional. Revista de Psicologia Universitas Tarraconensis, 12(1-2), 25-39.

Michie, S., \& Williams, S. (2003). Reducing work related psychological ill health and sickness absence: A systematic literature review. Occupational and Environmental Medicine, 60(1), 3-9.
Muñoz-Seco, E., Coll-Benejam, J. M., Torrent-Quetglas, M., \& Linares-Pou, L. (2006). Influencia del clima laboral en la satisfacción de los profesionales sanitarios. Aten Primaria, 37(4), 209-214.

Navarro-Reynoso, F., Hernández-Lamas, H., RobledoGalván, H., \& Romero-Palacios, T. (2008). Encuesta de satisfacción del personal de cinco servicios de un hospital general. Revista CONAMED, 12(3), 5-12.

Oliver, A., Tomás, J. M., \& Cheyne, A. (2005). Clima de seguridad laboral: naturaleza y poder predictivo. Revista de Psicología del Trabajo y de las Organizaciones, 21(3), 253-268.

Ortiz, J. A., \& Arias, F. (2009). El desgaste profesional (burnout) y calidad de vida laboral como predictores de la búsqueda de otro trabajo en profesionales de la salud y de la educación en el occidente de México. Ciencia y Trabajo, 34, 222-226.

Paredes, L. A., \& Paravic, T. (2006). Percepción de violencia de usuarios hospitalizados en los servicios clínicos de un hospital público de la Región de la Araucanía de Chile. Ciencia y Enfermería, 12(1), 39-51.

Peiró, J. M. (2004). El sistema de trabajo y sus implicaciones para la prevención de riesgos psicosociales. Universitas Psychologica, 3(2), 179-186.

Pérez, L., Soler, S., \& Díaz, L. (2009). Ambiente laboral en los policlínicos universitarios. Educación Médica Superior [Online], 23(2). Disponible en http://scielo.sld.cu/scielo.php?script=sci arttext\&pid=S0864-21412009000200004\&lng $=\mathrm{es} \& \mathrm{nrm}=\mathrm{iso} \& \mathrm{t} \operatorname{lng}=\mathrm{es}$

Paris, L., \& Omar, A. (2008). Predictores de satisfacción laboral en médicos y enfermeros. Estudos de Psicologia, 13(3), 233-244.

Pozo, C., Alonso, E., Hernández, S., \& Martos, M. (2005). Determinantes de la satisfacción laboral en trabajadores de la administración pública: el valor de las relaciones interpersonales en el lugar de trabajo. Ansiedad y Estrés, 11(2-3), 247-264.

Raigosa, D., \& Marín, B. (2010). Efficacy beliefs training: A proposal to reduce burnout and improve levels of engagement among employees. International Journal of Psychological Research, 3(2), 86-92.

Ramírez, M. (2008). Validación de una escala de Clima Organizacional en una muestra multiocupacional 
de la ciudad de Arica. Arica: Departamento de Filosofía y Psicología, Universidad de Tarapacá.

Ramírez, M., \& Zurita, R. (2010). Variables organizacionales y psicosociales asociadas al síndrome de burnout en trabajadores del ámbito educacional. Revista de la Universidad Bolivariana, 9(25), $515-$ 534.

Riveros, J., Berné, C., \& Múgica, J. M. (2010). Gestión y satisfacción en servicios de salud de Chile: contraste entre las percepciones de los funcionarios y los usuarios. Revista Médica de Chile, 138(5), 630-638.

Robles-García, M., Dierssen-Soto, T., Martínez-Ochoa, E., Herrera-Carral, P., Díaz-Mendi, A. M., \& Llorca-Díaz, J. (2005). Variables relacionadas con la satisfacción laboral: un estudio transversal a partir del modelo EFQM. Gaceta Sanitaria, 19(2), $127-134$

Salanova, M., \& Llorens, S. (2008). Estado actual y retos futuros en el estudio del burnout. Papeles del Psicólogo, 29(1), 59-67.

Salanova, M., \& Llorens, S. (2009). Exposición a la Tecnología de la Información y la Comunicación y su Relación con el engagement. Ciencia y Trabajo, 11(32), 55-62.

Salanova, M., \& Schaufeli, W. B. (2004). El engagement en los empleados: un reto emergente para la dirección de los recursos humanos. Estudios Financieros, 61(62), 109-138.

Salanova, M. \& Schaufeli, W. (2008). A cross-national study of work engagement as a mediator between job resources and proactive behaviour. The International Journal of Human Resource Management, 19(1), 116-131.

Salanova, M., Shaufeli, W. B., Llorens, S., Peiró, J. M. \& Grau, R. (2000). Desde el "burnout" al "en- gagement": una nueva perspectiva. Revista de Psicología del Trabajo y de las Organizaciones, 16(2), 117-134.

Santa Eulalia, J. M., \& Sampedro, B. A. (2012). Clima organizacional en instituciones de atención primaria de salud. Revista Médica Electrónica, 34 (5).

Schaufeli, W. B., \& Bakker, A. B. (2004). Job demands, job resources and their relationship with burnout and engagement: A multi-sample study. Journal of Organizational Behavior, 25(3), 293-315.

Schaufeli, W. B., Salanova, M., González-Romá, V., \& Bakker, A. (2002). The measurement of burnout and engagement: A confirmative analytic approach. Journal of Happyness Studies, 3(1), 71-92.

Segurado, A., \& Argulló, E. (2002). Calidad de vida laboral: hacia un enfoque integrador desde la Psicología Social. Psicothema, 14(4), 828-836.

Shu, M. Y., \& Kernohan, G. (2006). Dimensions of hospital nurses' quality working life. Journal of Advanced Nursing, 54(12), 120-131.

Sirgy, M. J., Efraty, D., Siegel, P., \& Lee, D. J. (2001). A new measure of quality of work life (QWL) based on need satisfaction and spillover theory. Social Indicators Research, 55(3), 241-302.

Taris, T. W., Peeters, M. C., Le Blanc, P. M., Schreurs, P. J., \& Shaufeli, W. B. (2001). From inequity to burnout: The role of job stress. Journal of Ocсupational Health Psychology, 6(4), 303-323.

Van Laar, D., Edwards, J. A., \& Easton, S. (2007). The Work-Related Quality of Life Scale for healthcare workers. Journal of Advanced Nursing, 60(3), 325-333.

Zuger, A. (2004). Dissatisfaction with medical practice. The New England Journal of Medicine, 350(1), 69-75. 
